\title{
Engineering the Personal Social Semantic Web
}

\author{
Fabian Abel and Geert-Jan Houben \\ Web Information Systems, Delft University of Technology \\ $\{$ f.abel,g.j.p.m.houben $\}$ @tudelft.nl
}

\begin{abstract}
In this tutorial, we discuss challenges and solutions for engineering the Personal Social Semantic Web, a Web where user modeling and personalization is featured across system boundaries. Therefore, we learn user modeling and personalization techniques for Social Web systems. We dive into engineering aspects of social tagging and microblogging services and examine appropriate modeling and mining techniques for these systems. We discuss Semantic Web and Linked Data principles that allow for linkage and alignment of distributed user data and show how system engineers can exploit the Social Semantic Web to personalize user experiences.
\end{abstract}

\section{Summary}

Social Web sites, such as Facebook, YouTube, Delicious, Flickr and Wikipedia, and numerous other Web applications, such as Google and Amazon, rely on implicitly or explicitly collected data about their users and their activities to provide personalized content and services. As these applications become more and more connected on the Web, a major challenge is to allow various applications to exchange, reuse, and integrate the user data from different sources. Such data comes in different flavors: user data such as user profiles, social networks, social tagging data, blogs, etc. as well as usage data like clickthrough data or query logs. The amount of user data available on the Web is tremendously growing so that sharing and mining these heterogeneous data corpora distributed on the Web is a non-trivial problem that poses several challenges to the Web engineering community.

A core challenge is to support people in overcoming the information overload on the Web. Here, adaptation and personalization are key strategies as people have individual demands and thus need individual support. However, understanding the personal demands of people is another non-trivial challenge. It requires appropriate solutions that allow for inferring and modeling the personal concerns, interests, preferences and other user characteristics. In this tutorial, we discuss user modeling and personalization within the context of today's Web sphere where Social Web systems foster user participation and where Semantic Web technologies provide means to engineer interoperable services.

This tutorial is composed of four modules: we give (i) an introduction into basic concepts and approaches for user modeling, adaptation and personalization (UMAP) on the Web and (ii) summarize methods and metrics that allow 
engineers and researchers to evaluate the quality of UMAP systems. We outline (iii) basic and advanced models and algorithms for implementing UMAP functionality in the context of Social Web systems and finally present (iv) strategies and techniques for engineering the personalized systems on the Social Web by leveraging Semantic Web technologies. In each module we give an overview of related work and recent trends, discuss selected models, algorithms and techniques in detail and provide hands-on examples.

Introduction to UMAP. We introduce basic user modeling techniques such as stereotyping or overlay user modeling, basic adaptation principles and personalization techniques. In particular, we summarize core principles of content-based and collaborative recommender systems and present Web mining methods.

Evaluation of UMAP systems. Evaluating the quality of user modeling and personalization often requires implicit or explicit user feedback which can be costly to obtain. We give an overview on evaluation strategies such as user studies and leave-n-out evaluation methods. Furthermore, we outline useful metrics, significance tests and present examples on evaluating UMAP functionality on the Web.

UMAP on the Social Web. Here, we discuss models and techniques for inferring user interests in Social Web systems and exploiting user profiles for personalization such as personalized search or social recommender systems. Moreover, we examine the challenges and opportunities of cross-system user modeling and personalization.

Engineering the Personal Social Semantic Web. Given techniques learnt before, we investigate principles for personalizing user experiences in the Social Semantic Web. We discuss Linked Data principles, techniques for connecting online accounts of users - including useful vocabularies, tools and services - and approaches for aligning user data originating from different sources. Based on these solutions, we explore architectures for cross-system user modeling and personalization, methods and protocols for ensuring trust and privacy and outline future perspectives for building a Personal Social Semantic Web.

Some parts of this tutorial are based on 2]. Supplemental material, slides and references for this tutorial are publicly available via the supporting website [1].

\section{References}

1. Abel, F., Houben, G.J.: Engineering the Personal Social Semantic Web - Supporting Website (2011), http://wis.ewi.tudelft.nl/icwe2011/tutorial/

2. Abel, F., Herder, E., Houben, G.J., Henze, N., Krause, D.: Cross-system User Modeling and Personalization on the Social Web. In: User Modeling and User-Adapted Interaction (UMUAI), Special Issue on Personalization in Social Web Systems, vol. 22(3), pp. 1-42 (2011) 